\title{
Draft genome sequence of a multidrug-resistant Acinetobacter baumannii ST15 (CC15) isolated from Brazil
}

\author{
Thiago Pavoni Gomes Chagas ${ }^{1 /+}$, Melise Chaves Silveira², Rodolpho Mattos Albano ${ }^{3}$, \\ Ana Paula D'Alincourt Carvalho-Assef' ${ }^{1}$, Marise Dutra Asensi ${ }^{1}$
}

\begin{abstract}
${ }^{1}$ Fundação Oswaldo Cruz, Instituto Oswaldo Cruz, Laboratório de Pesquisa em Infecção Hospitalar, Rio de Janeiro, RJ, Brasil
${ }^{2}$ Fundação Oswaldo Cruz, Instituto Oswaldo Cruz, Laboratório de Biologia Computacional e Sistemas, Rio de Janeiro, RJ, Brasil

${ }^{3}$ Universidade do Estado do Rio de Janeiro, Instituto de Biologia Roberto Alcântara Gomes, Departamento de Bioquímica, Rio de Janeiro, RJ, Brasil
\end{abstract}

Acinetobacter baumannii is an important pathogen frequently associated with nosocomial outbreaks around the world. In Brazil, A. baumannii has become particularly problematic because of its prevalence and the carbapenems resistance. Here, we report the draft genome sequence of a multidrug-resistant A. baumannii (ST15/CC15) isolated in 2009 from the state of Espirito Santo (Southeast Brazil). We observed important resistance determinant genes in an estimated genome size of 4,102,788 bp with 3,862 predicted coding regions. A detailed report of the genomic data analysis might help to understand the specific features of highly successful strains belonged to a relevant complex clonal in different Brazilian geographical regions.

Key words: carbapenem-resistance - OXA-23 - Acinetobacter baumannii - Brazil - genome sequence

Acinetobacter baumannii is an important Gram-negative pathogen frequently associated with nosocomial outbreaks worldwide and highly capable of surviving and spreading in the hospital environment. This pathogen may present high rates of multi-drug resistance including aminoglycosides and fluoroquinolones. Carbapenems usually have good potency against $A$. baumannii, but carbapenem resistance has been increasingly reported in several countries during the last decade (Migliavacca et al. 2013). Carbapenem-hydrolysing class D $\beta$-lactamases of the oxacilinases are the most important cause of carbapenem resistance in A. baumannii around the world (Zarrilli et al. 2013).

In Brazil, A. baumannii has become particularly problematic because of its prevalence and the carbapenems resistance, usually related to oxacilinases, mostly involving OXA-23 producers (Carvalho et al. 2009). Previous study reported the dissemination of two major clusters, clonal complexes (CC) 15 and 79, of multidrug-resistant $A$. baumannii in different Brazilian states harbouring bla ${ }_{\mathrm{OXA}-23}$ gene. The $\mathrm{CC} 15$ was described in nine Brazilian states [Alagoas, Distrito Federal, Espírito Santo (ES), Goiás, Minas Gerais, Rio de Janeiro, Rio Grande do Norte, Santa Catarina and Mato Grosso do Sul] belonging to the four different geographical regions of the country (Chagas et al. 2014).

doi: 10.1590/0074-02760150158

Financial support: CNPq, FAPERJ, IOC (PAPES-FIOCRUZ)

+ Corresponding author: thiagopavoni@gmail.com

Received 21 April 2015

Accepted 15 June 2015
This report announces the draft genome sequence of A. baumannii CCBH5233 isolated in 2009 from a hospitalised patient in ES (Southeast Brazil) and deposited in the Culture Collection of Hospital-Acquired Bacteria (CCBH) located at the Hospital Infection Research Laboratory (Oswaldo Cruz Institute/Oswaldo Cruz Foundation). This strain displayed a multidrug resistance profile, which included resistance to ciprofloxacin, piperacillin/tazobactam, ampicillin/sulbactam, ceftazidime, cefepime, amikacin, sulfamethoxazole-trimethoprim, meropenem and imipenem, with the exception of polymyxin B. According to polymerase chain reaction (PCR) analysis (Higgins et al. 2013), A. baumannii CCBH5233 has bla ${ }_{\text {OXA-51-like }}$, originally intrinsic to $A$. baumannii and bla Previous multilocus sequence typing (MLST) analysis (Diancourt et al. 2010) showed that the strain belongs to sequence type (ST) 15/CC15 (according to MLST scheme developed by Pasteur Institute) (Chagas et al. 2014).

Genomic DNA was extracted using QIAamp DNA Blood Mini Kit (Qiagen, Germany) and sequenced using an Illumina MiSeq sequencing platform (Illumina Inc, USA). A genomic library was constructed by transposon tagmentation with the Nextera XT DNA Sample Prep kit (Illumina Inc). The data were filtered and trimmed to a Phred score of $>20$ and were assembled de novo using the Velvet algorithms (Zerbino \& Birney 2008). Seventy-six contigs were generated and genome annotation was performed on the Rapid Annotation using System Technology (RAST) v.2.0 (available from rast.nmpdr. org/). By RAST, 3,862 coding sequences were annotated from the genome with estimated size of 4,102,788 bp. According to tRNAScan-SE (Schattner et al. 2005) and RNAmmer (Lagesen et al. 2007) analysis we identified 66 tRNAs and eight rRNAs, respectively. A. baumannii contigs were analysed using ResFinder 2.1 (available from cge.cbs.dtu.dk/services/ResFinder/) which identifies acquired antimicrobial resistance genes in total or partial 
sequenced isolates of bacteria (Zankari et al. 2012). Using this approach, we observed resistance genes to betalactams [ bla $_{\text {OXA-51 }}$ (LAXF01000004; 277,103-277,927 bp) bla $_{\text {OXA-23 }}\left(\right.$ LAXF01000049; 9,858-10,679 bp) and $b^{\prime} a_{\text {TEM-1- }}$, (LAXF01000047; 5,481-6,341 bp)], to aminoglycosides [aphA(6) (LAXF01000061; 273-1,052 bp) and aac(3)-IIa (LAXF01000047; 4,538-5,339 bp)] and to sulphonamide [sul2 (LAXF01000056; 2,580-3,395 bp)].

In order to confirm the result obtained by traditional PCR-based MLST, A. baumannii contigs were analysed by MLST 1.7 server of Center for Genetic Epidemiology (available from cge.cbs.dtu.dk/services/MLST/) (Larsen et al. 2012). The MLST 1.7 results showed that CCBH5233 belongs to ST15/CC15 (MLST scheme developed by Pasteur Institute) and ST236/CC104 (MLST scheme developed by University of Oxford). Additional molecular typing was performed using Acinetobacter baumannii Sequence Typing server (available from hpabioinformatics.org.uk/AB/ab_type1.php) by searching and comparing the regions of ompA, csuE and bla ${ }_{\mathrm{OXA}-51}$ (Turton et al. 2007). Using this additional typing, our strain was defined as ST502.

In summary, we reported whole-genome sequencing of the A. baumannii ST15 (CC15) harbouring bla ${ }_{\mathrm{OXA}-23}$ and a detailed genomic analysis will be provided in future studies. In the context of resistance characteristics, genes relevant to Acinetobacter could be identified by whole genome sequencing approaches. A detailed report of the genomic data analysis might help to understand the specific features of successful strains belonging to an important complex clonal in Brazil.

This Whole Genome Shotgun project has been deposited at DNA DataBank of Japan/European Molecular Biology Laboratory/GenBank under the accession LAXF00000000. The version described in this paper is version LAXF01000000.

\section{REFERENCES}

Carvalho KR, Carvalho-Assef APD, Peirano G, Santos LC, Pereira MJ, Asensi MD 2009. Dissemination of multidrug-resistant Acinetobacter baumannii genotypes carrying $b l a_{\text {OXA-23 }}$ collected from hospitals in Rio de Janeiro, Brazil. Int J Antimicrob Agents 34: 25-28.
Chagas TP, Carvalho KR, de Oliveira Santos IC, Carvalho-Assef AP, Asensi MD 2014. Characterization of carbapenem-resistant Acinetobacter baumannii in Brazil (2008-2011): countrywide spread of OXA-23-producing clones (CC15 and CC79). Diagn Microbiol Infect Dis 79: 468-472.

Diancourt L, Passet V, Nemec A, Dijkshoorn L, Brisse S 2010. The population structure of Acinetobacter baumannii: expanding multiresistant clones from an ancestral susceptible genetic pool. PLOS ONE 5: e10034.

Lagesen K, Hallin P, Rødland EA, Staerfeldt HH, Rognes T, Ussery DW 2007. RNAmmer: consistent and rapid annotation of ribosomal RNA genes. Nucleic Acids Res 35: 3100-3108.

Higgins PG, Pérez-Llarena FJ, Zander E, Fernández A, Bou G, Seifert H 2013. OXA-235, a novel class D $\beta$-lactamase involved in resistance to carbapenems in Acinetobacter baumannii. Antimicrob Agents Chemother 57: 2121-2126.

Larsen MV, Cosentino S, Rasmussen S, Friis C, Hasman H, Marvig RL, Jelsbak L, Sicheritz-Pontén T, Ussery DW, Aarestrup FM, Lund O 2012. Multilocus sequence typing of total-genome-sequenced bacteria. J Clin Micobiol 50: 1355-1361.

Migliavacca R, Espinal P, Principe L, Drago M, Fugazza G, Roca I, Nucleo E, Bracco S, Vila J, Pagani L, Luzzaro F 2013. Characterization of resistance mechanisms and genetic relatedness of carbapenem-resistant Acinetobacter baumannii isolated from blood, Italy. Diagn Microbiol Infect Dis 75: 180-186.

Schattner P, Brooks AN, Lowe TM 2005. The tRNAscan-SE, snoscan and snoGPS web servers for the detection of tRNAs and snoRNAs. Nucleic Acids Res 33: 686-689.

Turton JF, Gabriel SN, Valderrey C, Kaufmann ME, Pitt TL 2007. Use of sequence-based typing and multiplex PCR to identify clonal lineages of outbreak strains of Acinetobacter baumannii. Clin Microbiol Infect 13: 807-815.

Zankari E, Hasman H, Cosentino S, Vestergaard M, Rasmussen S, Lund O, Aarestrup FM, Larsen MV 2012. Identification of acquired antimicrobial resistance genes. $J$ Antimicrob Chemother 67: 2640-2644.

Zarrilli R, Pournaras S, Giannouli M, Tsakris A 2013. Global evolution of multidrug-resistant Acinetobacter baumannii clonal lineages. Int J Antimicrob Agents 41: 11-19.

Zerbino DR, Birney E 2008. Velvet: algorithms for de novo short read assembly using de Bruijn graphs. Genome Res 18: 821-829. 